Leicester, Leicestershire and Rutland, the real life experiences of healthcare professionals and surviving relatives, have not been previously documented locally, and are only in limited detail in academic literature. This project was conducted as part of an MSc in Palliative Care.

Aim To interview key stakeholders, including healthcare professionals and surviving relatives, regarding their experiences of prescribing, administering, dispensing and observing $\mathrm{AM}$ at the EOL.

Method Semi-structured interviews were conducted as individuals or in small groups, with both healthcare professionals and relatives. These were: Community Palliative Care Nurses (5), Hospice at Home Nurses (4), District Nurses (1), General Practitioners (3), Community Pharmacists (1), and Relatives (2). Qualitative thematic analysis of interviews was conducted identifying clusters of themes, themes and sub-themes.

Results There were two main clusters of themes; Decision points in AM; and Goals of treatment. There were nine themes which all influenced the main clusters which included; patient experience; family experience, emotions; finances; improvements; multidisciplinary working; decision making service delivery; and standardised systems. Each theme had promoting and inhibiting factors, which would affect the Decision Points and Goals of Treatment. Overall, standardised systems were felt to have improved the Goals of Treatment. Variations in service delivery, decision making, MDT working and financial concerns all potentially adversely affect the Goals of Treatment. All interviewees indicated one key element, the importance of managing symptoms at the EOL: 'The patient died peacefully at home'.

Conclusions Decisions about AM, and meeting the Goals of Treatment, are part of a complex healthcare system.

Effective EOL symptom management in the community is important for patients, relatives and carers, and healthcare professionals, and AM play an important role to achieve this.

Further research is indicated to interview greater numbers of key stakeholders to expand on this work.

\section{P-122 CONSISTENT COUNTYWIDE GUIDANCE AROUND ANTICIPATORY MEDICATIONS USED DURING THE DYING PHASE}

\begin{abstract}
${ }^{1}$ Jane Gibbins, ${ }^{1}$ Kirsty Scott, ${ }^{2}$ Michael Thomas, ${ }^{3}$ Angela Carey, ${ }^{3}$ Saul Ridley, ${ }^{4}$ Rachel Newman, ${ }^{4}$ Elizabeth Thomas, ${ }^{4}$ Angela Hart, ${ }^{1}$ Carolyn Campbell, ${ }^{1}$ Melanie Huddart, ${ }^{1}$ Deborah Stevens, ${ }^{3,4}$ Joanne Smith. ${ }^{1}$ Cornwall Hospice Care, St Austell, UK; ${ }^{2}$ St Luke's Hospice, Plymouth, UK; ${ }^{3}$ Peninsula Community Health, St Austell, UK; ${ }^{4}$ Royal Cornwall Hospital Trust, Truro, UK
\end{abstract}

\subsection{6/bmispcare-2017-hospice. 147}

Background Nationally, there is awareness of the need to improve symptom control for patients at end of life (National VOICES survey).

Aim Dissemination of unified Anticipatory Prescribing Guidance (APG) (incorporating opioid conversion chart, and Hospice 24 hour/7-days-a-week-advice-line-number) to all healthcare settings across Cornwall, backed by a programme of education.

Methods APG developed and rolled out with teaching by specialist palliative care (SPC) teams throughout the county and incorporating attendees' experience to inform the evolution of the project, using Quality Improvement (QI) methodology. Participants were asked to complete a paper questionnaire at the time of teaching to assess changes in knowledge, and an electronic questionnaire 12 weeks after the teaching to capture the impact of such teaching on care of the dying. Quantitative data was analysed using the non-parametric-WilcoxonMatched-Pairs. Qualitative data was analysed using thematic analysis until saturation was achieved.

Settings All healthcare sectors; acute and community hospitals, hospices, nursing homes, GPs, district nursing services, secure dementia units and ambulance service.

Results 990 healthcare professionals (HCPs) were taught. There was a statistically significant shift in median knowledge scores ( $p$ value $<0.001$ ). HCP perceived there was an improvement of symptom control for the dying in clinical practice. Five main themes emerged from the qualitative data; common guidance, improved knowledge and assessment of symptomcontrol and opioid conversions, advice 24/7-feeling safe, recognising dying and considering what is important to patients, and confidence building.

Conclusion It has been possible to roll out unified APG to a wide range of HCPs to improve their knowledge and confidence. In clinical practice, HCPs perceive it enables improved care to patients in the dying phase by improving symptom control. HCPs described their practice as safer and more efficient. Healthcare professionals formally and informally described the positive impact of having specialist palliative care/hospice-advice-24/7 to back up the guidance on the ground.

\section{P-123 THE RIGHT FORMULA FOR COST EFFECTIVE PRESCRIBING IN PALLIATIVE CARE}

Kate Campbell, Katrin Little, Paula Powell. Willowbrook Hospice Merseyside UK

10.1136/bmjspcare-2017-hospice. 148

In 2016 Willowbrook Hospice had to make the change to an alternative supplier of drugs and drug costs increased substantially. This additional cost is picked up by the CCGs commissioning services from the hospice.

This resulted in a review of prescribing in the inpatient unit and outpatient clinics. Medical and nursing staff were quite often unaware of the cost of medications and prescribing was driven by what was familiar or in stock. We found that there were significant potential cost savings that could be made through better management of stock, brand and cost effective prescribing.

This poster describes the development of a hospice formulary and the initial impact of this on prescribing patterns and costs.

The first chapter of the formulary covered laxative prescribing - this has resulted in a $70 \%$ reduction in cost each month amounting to approximately $£ 7000$ per year. This has been achieved without any adverse impact on patients.

Further chapters on opioids and gastrointestinal medications are about to be added and we intend to continue this development alongside better education for staff on cost effective prescribing. 